\title{
Common Benign Conditions of the Petrous Apex in the Pediatric Population
}

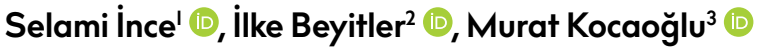 \\ 'Clinic of Radiology, Beytepe Murat Erdi Eker State Hospital, Ankara, Turkey \\ ${ }^{2}$ Department of Pediatrics, Near East University School of Medicine, Nicosia, Cyprus \\ ${ }^{3}$ Department of Radiology, Near East University School of Medicine, Nicosia, Cyprus \\ ORCID IDs of the authors: S.i. 0000-0002-66II-4962; i.B. 0000-0002-7758-9015; M.K. 0000-0002-4674-8634.
}

Cite this article as: Ince S, Beyitler I, Kocaoğlu M. Common Benign Conditions of the Petrous Apex in the Pediatric Population. Cyprus J Med Sci 2018; 3(3): 173-7.

\begin{abstract}
Petrous apex lesions are rare. However, a wide number of entities may be seen during radiological studies of the head and neck. In addition to malignant pathologies, a variety of benign lesions can be discovered within this structure, such as cholesterol granuloma cholesteatoma, cephalocele, mucocele, apical petrositis, and Langerhans cell histiocytosis. In addition to these lesions, a number of normal imaging variants may complicate the diagnosis. An understanding of the patterns and extent of disease processes of the petrous apex facilitates diagnosis and staging. Plain radiography, high-resolution computed tomography, and magnetic resonance imaging with diffusion-weighted sequences can be used in the assessment of petrous apex.
\end{abstract}

Keywords: CT, MRI, pediatric, petrous apex, temporal bone

\section{INTRODUCTION}

The petrous apex is the pyramidal shape medial projection of the temporal bone anteromedial to the inner ear and lateral to the petro-occipital fissure. It is subdivided by the internal auditory canal into larger anterior and smaller posterior segments (I). Petrous apex lesions are rare; however, a wide number of entities may be seen during radiological studies of this region incidentally for an unrelated disease. In addition, pseudolesions involving the petrous apex may make the diagnosis difficult and cause unnecessary treatment. Owing to its complex anatomical localization imaging, plain radiography, high-resolution computed tomography (HRCT), and magnetic resonance (MR) imaging with diffusion-weighted (DW) sequences play an important role in the assessment of petrous apex $(2,3)$. The aims of this pictorial essay were to describe the pseudolesions involving the petrous apex, to summarize the benign pathologies, and to recognize the patterns and imaging features of diseases that may involve the petrous apex.

\section{Normal Anatomic Variants of the Petrous Apex}

Mostly, the petrous apex is non-aerated and composed of dense bone and bone marrow (Figure I). In up to $30 \%$ of the cases, variable degree of aeration of the petrous apex occurs. Approximately $4 \%-7 \%$ of the pneumatized petrous
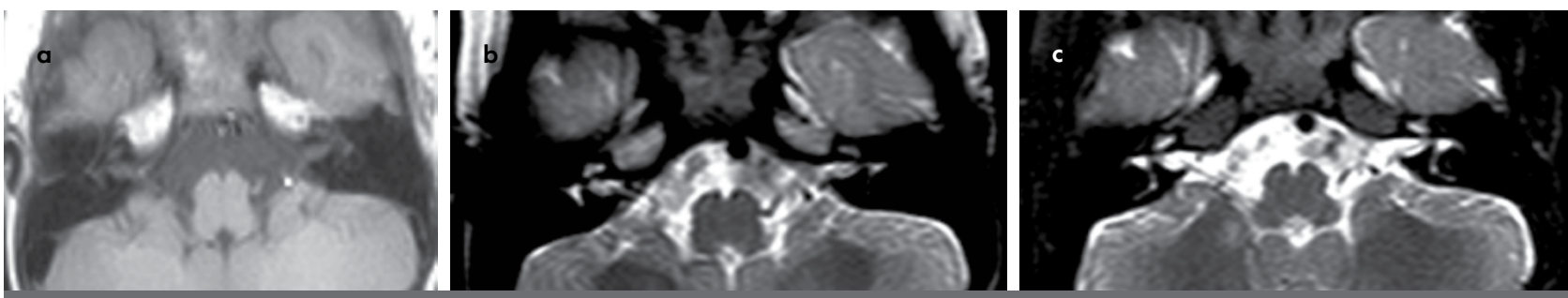

FIGURE I. a-c. Non-pneumatized petrous apex. Bone marrow of both petrous apices reveals high $\mathrm{TI}$ (a) and intermediate T2 signal (b) that significantly decreased on fat-suppressed T2-weighted image (c). 
bones are asymmetric (4). When the pneumatization is not symmetric, the non-pneumatized petrous apex may be misinterpreted as pathologic (Figure 2). Absence of mass effect and close observation of the signal changes with fat-suppressed sequences prevent misinterpretation as cholesteatoma, effusion, or cholesterol granuloma. In addition, CT can easily visualize normal cortex or bony trabecula without mass effect. Asymmetric aeration is associated with asymmetric fatty marrow in the petrous apex. Typically, signal intensity of fatty marrow follows the signal of retro-orbital fat in all MR sequences. Fat-suppressed MR sequences or CT can confirm the normal fatty marrow $(2,3,5)$.

\section{Cephalocele}

By definition, petrous apex cephalocele is the cystic expansion and herniation of the posterolateral portion of Meckel's cave into the superomedial portion of petrous apex. They are cerebrospinal fluid (CSF)-filled cystic structures. Except for fibers of the fifth cranial nerve, petrous apex cephalocele does not contain other neuronal tissues including the brain parenchyma. This entity is usually regarded as an incidental finding and believed to occur when CSF pressure caused over petrous apex roof dehiscence. On imaging, cephaloceles follow the CSF density or intensity on CT and MR imaging, respectively (Figure 3) $(1,3,6)$.
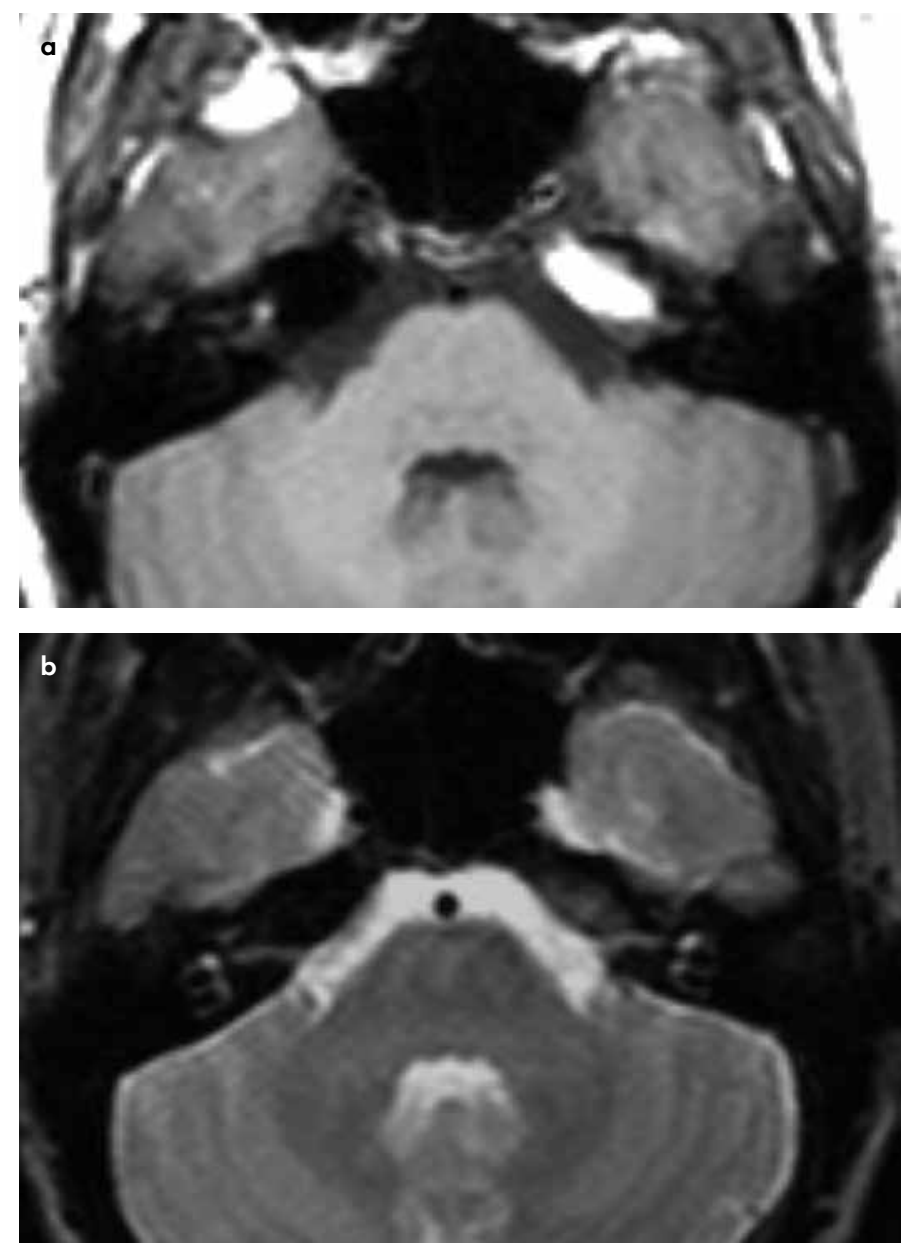

FIGURE 2. a, b. Asymmetric petrous apex. TI-weighted axial MR image shows high signal intensity of the left petrous apex suggesting cholesterol granuloma (a). T2-weighted axial MR image, however, reveals that these appearances follow the subcutaneous fat signal and consistent with non-pneumatized petrous apex (b).

\section{Effusion and Petrous Apicitis}

Petrous apex cells have communication with the middle ear; therefore, they are involved with similar disorders including inflammation and obstruction. Following a middle ear infection, a sterile fluid accumulation can be seen in petrous apex air cells; however, in the antibiotic era, extension of middle ear infection into the pneumatized petrous apex is rare. In addition to secondary involvement, isolated/primary mucosal inflammation and fluid retention can also occur due to viral agents and allergens. Isolated petrous apex effusion is a relatively frequent incidental imaging finding, and usually no follow-up or treatment is necessary (4). On imaging, fluid accumulation follows the CSF density and intensity on CT and MR imaging, respectively, with the preservation of internal septations (Figure 4). However, TI signal can be intermediate or high depending on the protein
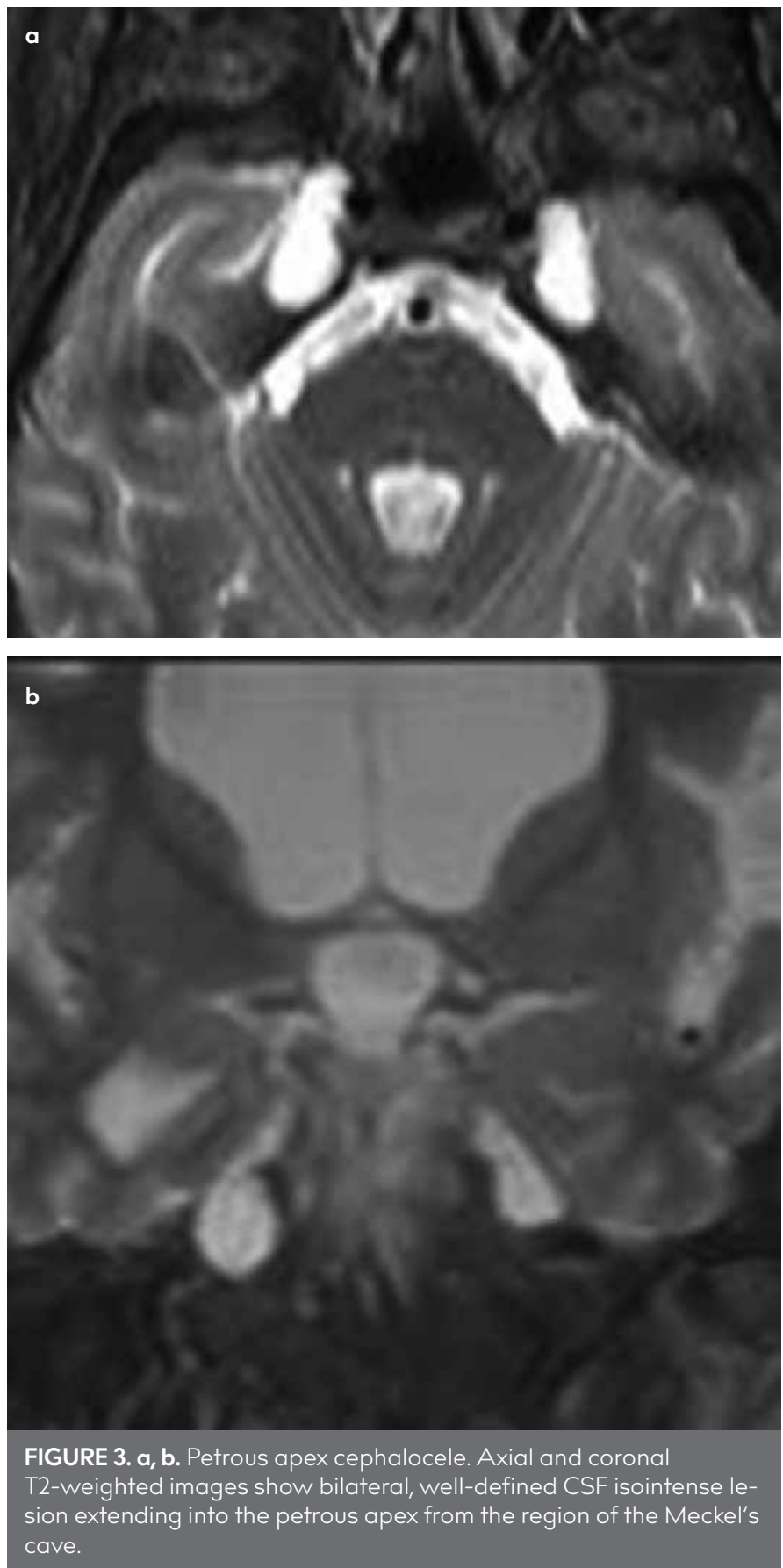

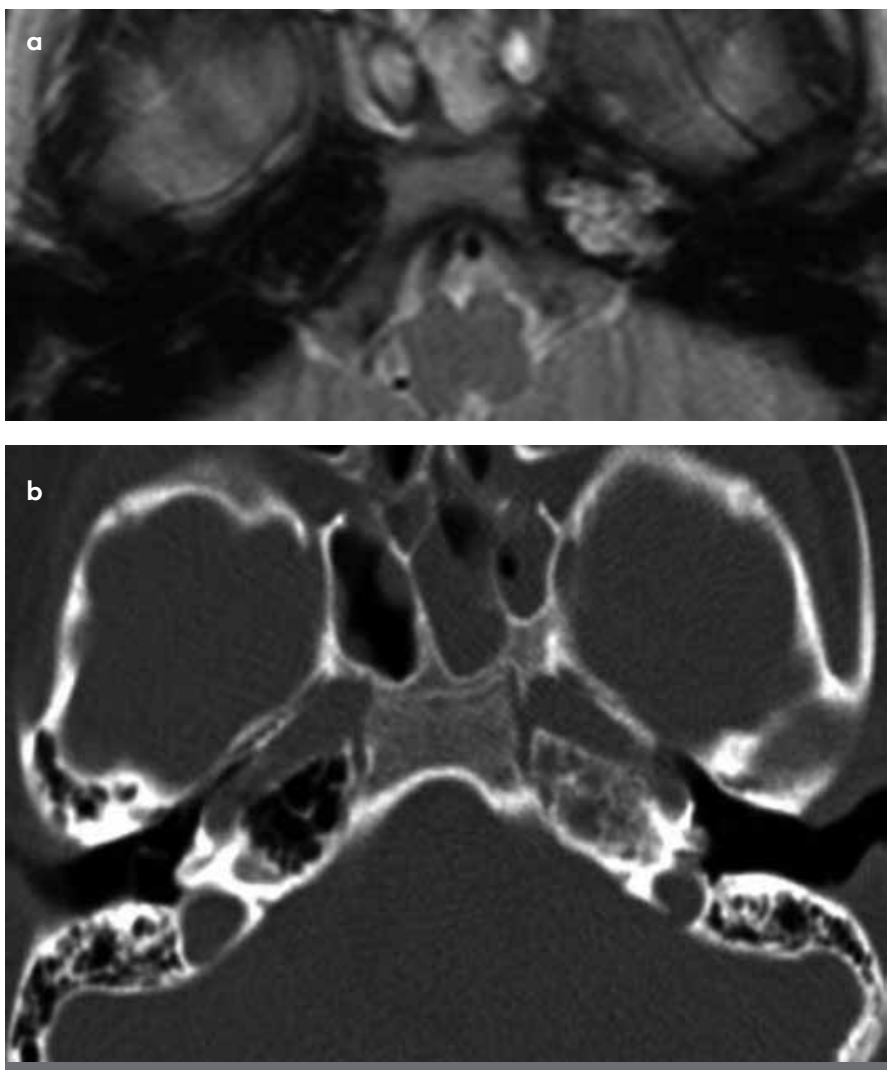

FIGURE 4. a, b. Petrous apex effusion. On T2-weighted image, there is a high T2 lesion in the left petrous apex (a). HRCT of the temporal bone reveals opacification of the left petrous apex with the preservation of air cells (b). Absence of expansion and presence of trabecular thinning suggest the diagnosis of effusion.

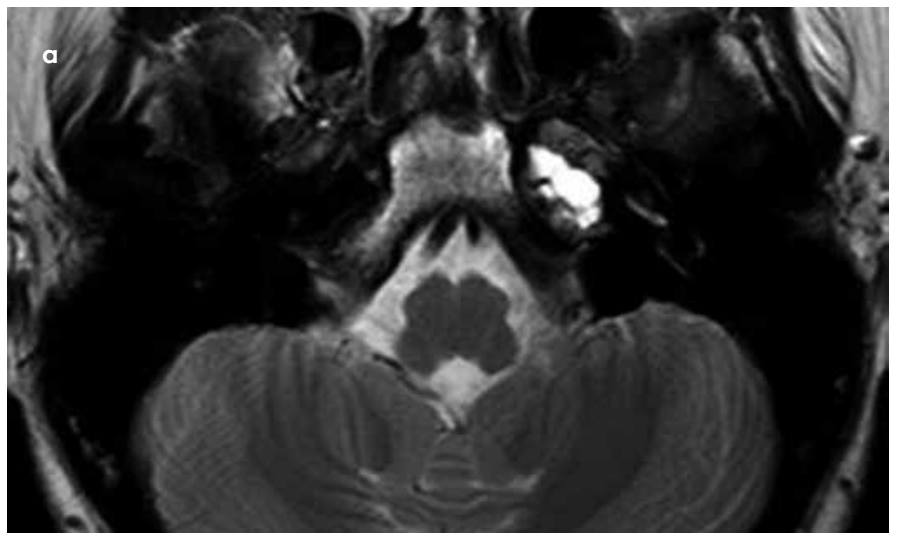

c

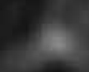

content of the fluid, which can cause confusion with cholesterol granuloma and mucocele. Lack of contrast enhancement and trabecular disruption can be helpful in differentiation from these lesions (5).

\section{Mucocele}

Mucocele is a rare inflammation that is believed to result from air cell obstruction. Accumulation of mucoid material within the obstructed air cells causes expansion, remodeling, and destruction of the bony septations. This disorder rarely primarily occurs in the petrous apex; instead, it usually extends from the adjacent structures. CT of the temporal bone reveals opacification of the air cells that is indistinguishable from cholesterol granuloma. Mucoceles have high T2 signal intensity with variable TI signal on MR imaging $(3,7,8)$. It can also show peripheral enhancement without restriction in diffusion (Figure 5) (5).

\section{Cholesterol Granuloma}

Cholesterol granuloma is the most frequent adult petrous apex disorder that may occur in the mastoid, middle ear, and petrous apex; however, it is an uncommon petrous apex lesion in children. Its cause is unknown and believed to occur as an air cell obstruction, followed by mucosal edema, hemorrhage, inflammatory reaction, and bony expansion. Incidental small petrous apex cholesterol granulomas can be found on imaging studies that were performed for other symptoms. These asymptomatic lesions do not need any intervention until they progressively enlarge and cause symptoms. CT demonstrates an expansile, well-defined mass within the petrous apex with cortical thinning. Cholesterol granulomas classically have high signal intensity on both TI- and T2-weighted MR sequences
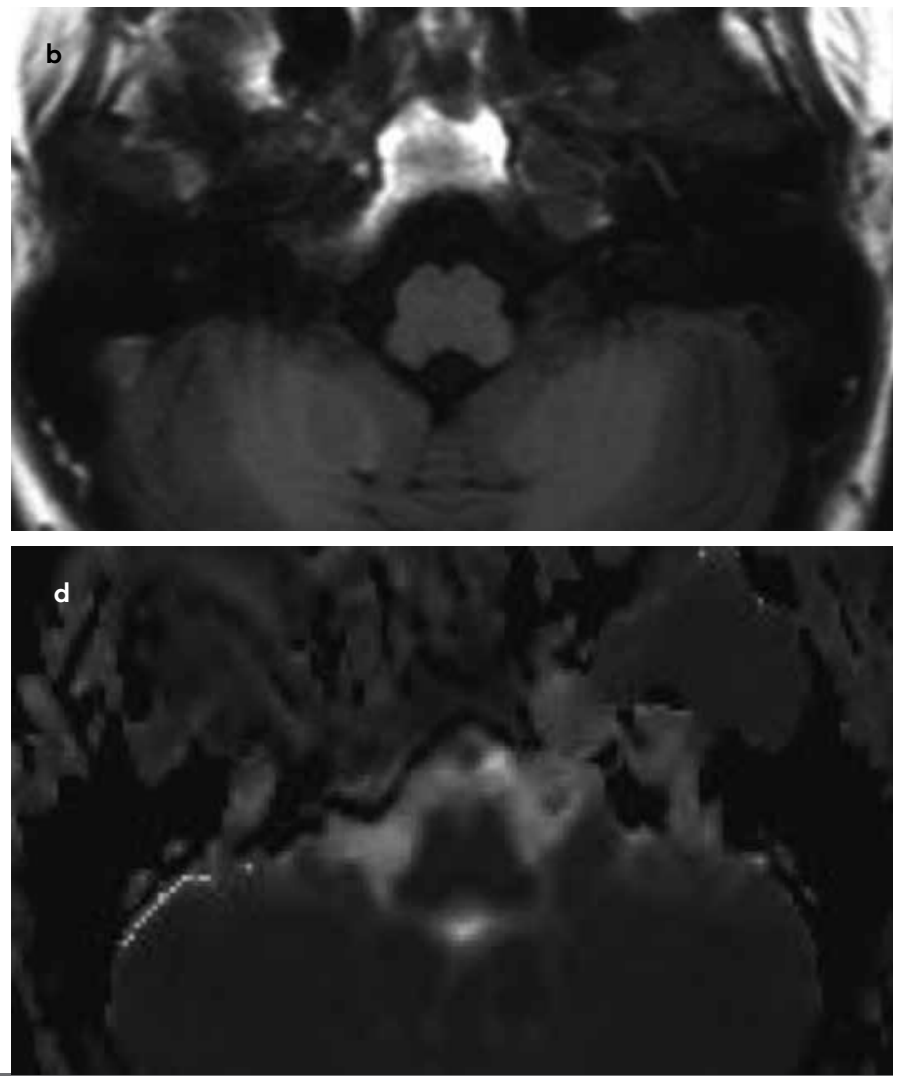

FIGURE 5. a-d. Petrous apex mucocele. Left petrous apex demonstrates mild expansion and high T2 (a) and low to intermediate signal intensity on TI-weighted image (b). The bl000 DW image (c) and corresponding ADC map (d) show that diffusion is not restricted. 

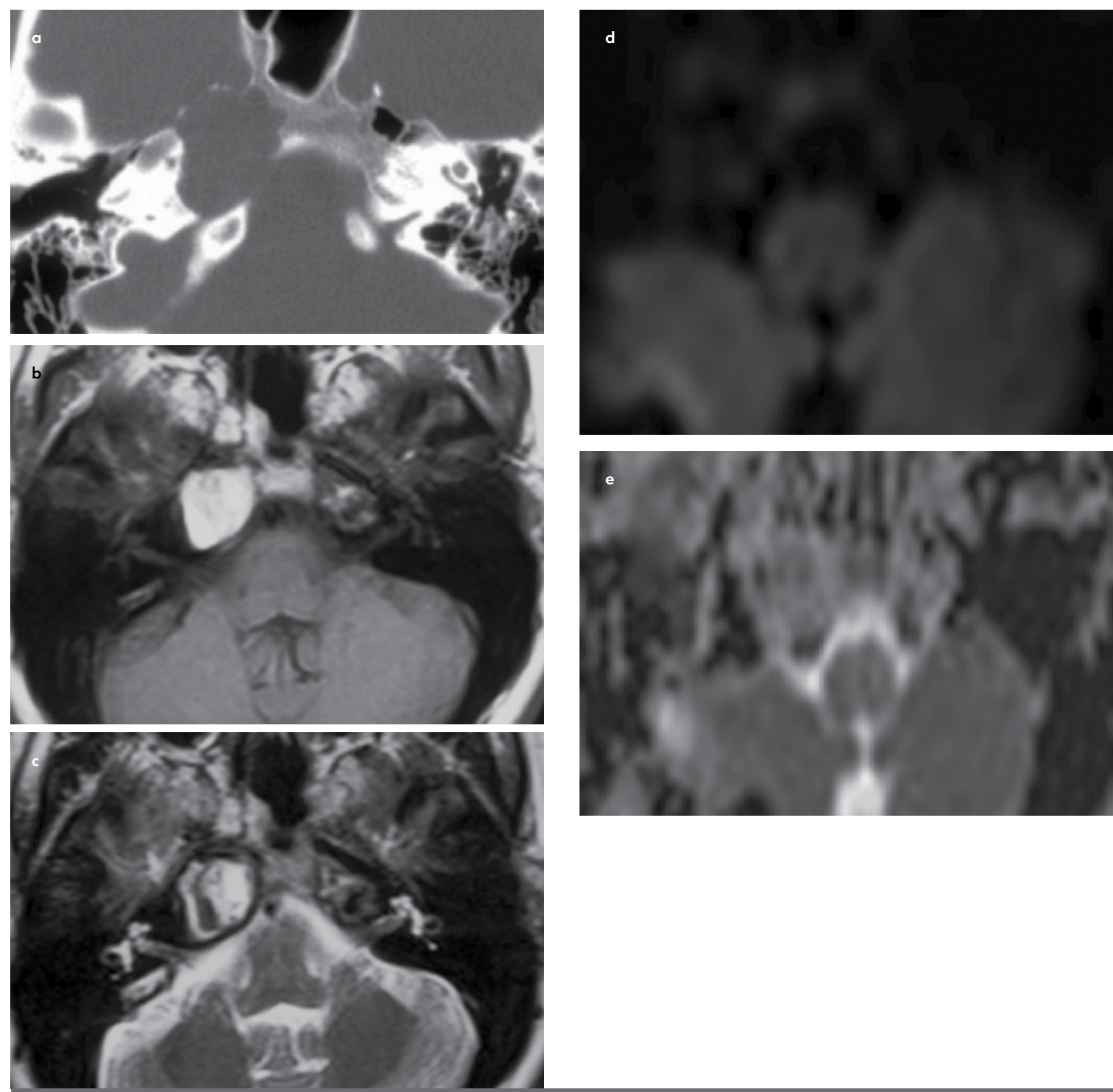

FIGURE 6. a-e. Cholesterol granuloma. An axial CT scan (a), TI- (b), and T2-weighted axial MR images (c) demonstrate expanded right petrous apex. The lesion shows high TI and high T2 signal intensity consistent with cholesterol granuloma. The bl000 DW image (d) and corresponding ADC map (e) show no restriction in diffusion. The dark peripheral rim is due to hemosiderin.

due to accumulation of blood products and proteinaceous debris (Figure 6). They also stay hyperintense after fat-suppressed MR sequences (8).

\section{Cholesteatoma}

Cholesteatoma or epidermoid cyst is a cystic mass-like lesion originating from the aberrant ectoderm trapped within the petrous apex. It can be seen at any part of the temporal bone including petrous apex. On imaging, they are expansile lesions with variable bone destruction. Typically, they have high T2- and low TI-weighted signal on conventional MR imaging sequences. Diffusion restriction is characteristic and helpful for distinguish- ing from mucocele (9). Half-Fourier acquisition single-shot turbo spin-echo DW imaging has high sensitivity and specificity in primary diagnosis and follow-up of possible residual/recurrence following surgery (10). They also may show subtle peripheral contrast enhancement (Figures 7, 8) (5).

\section{CONCLUSION}

Imaging plays a pivotal role in assessing petrous apex lesions and normal variants. An understanding of the variants, pitfalls, and patterns of disease processes of the petrous apex facilitates diagnosis and staging. HRCT and MR imaging including DW sequences allow detailed evaluation of this region. 


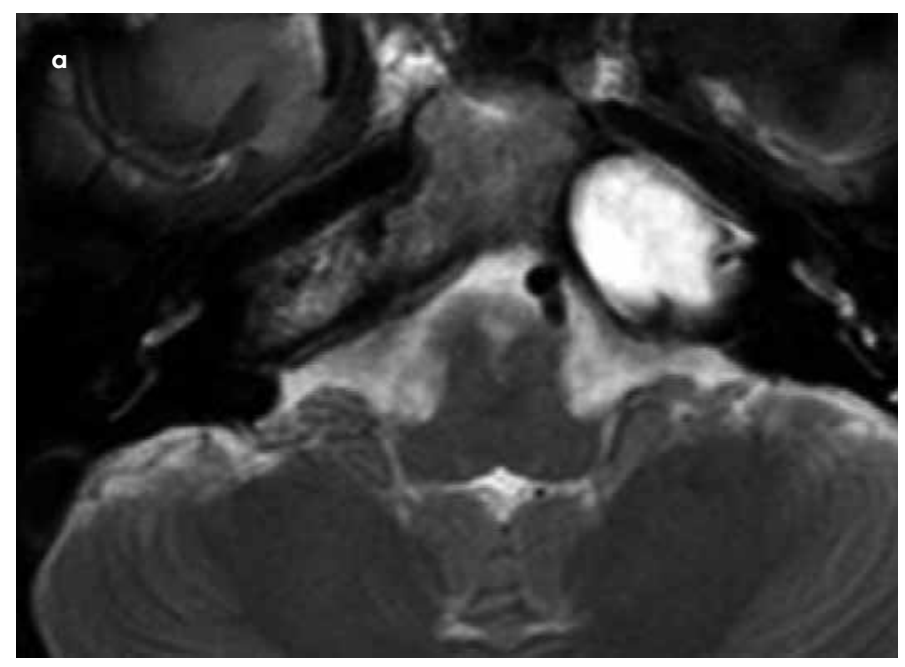

b

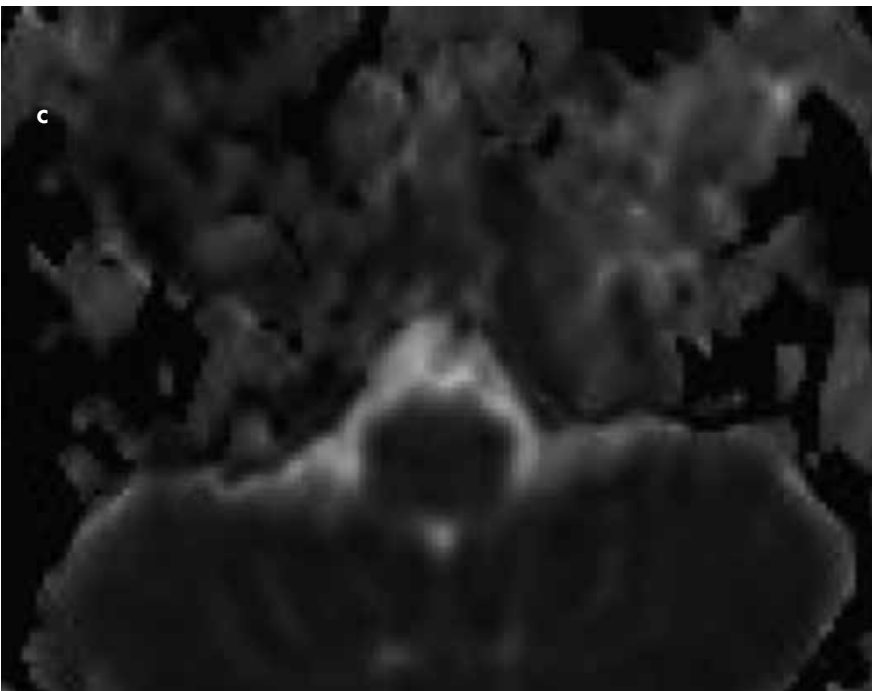

FIGURE 7. a-c. Cholesteatoma. On T2-weighted image (a), there is a well-defined, hyperintense expansile lesion in the left petrous apex. The bl000 DW image (b) and corresponding ADC map (c) show characteristic restriction in diffusion.

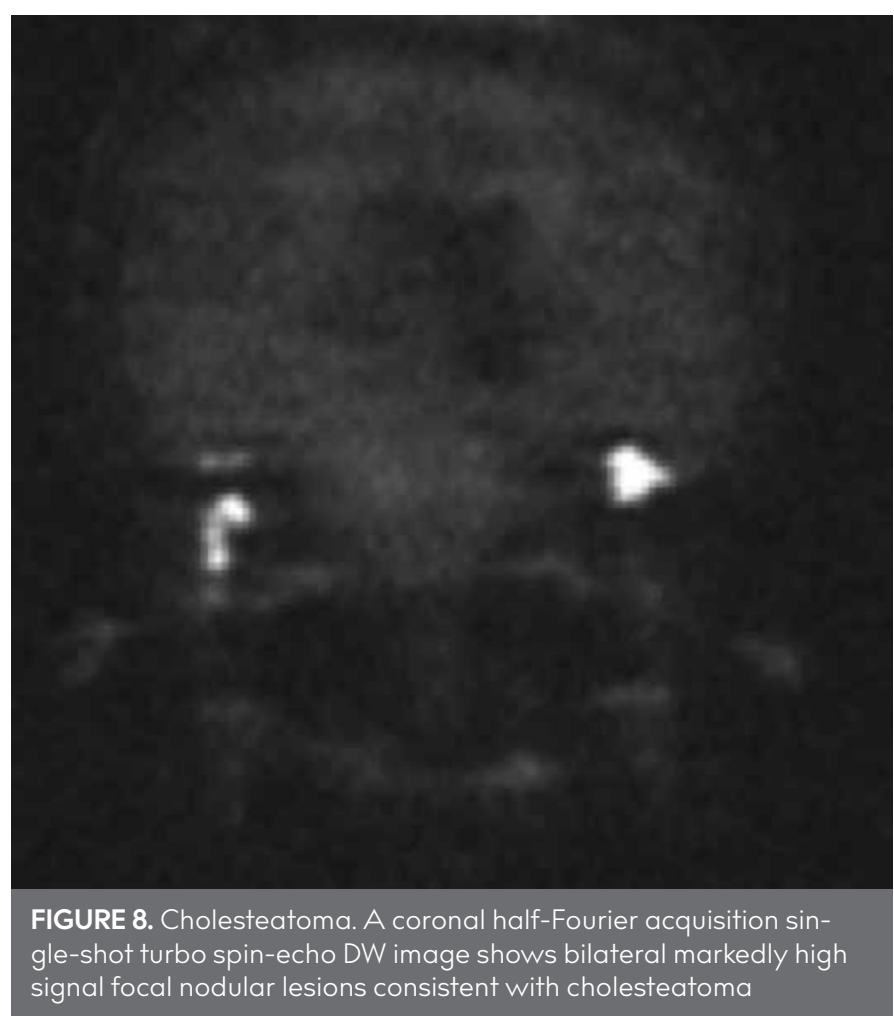

Peer-review: Externally peer-reviewed.

Author Contributions: Concept - S.I., I.B., M.K.; Design - S.I., I.B., M.K.; Supervision - M.K.; Data Collection and/or Processing - S.I., I.B., M.K..; Analysis and/or Interpretation - S.I., I.B., M.K.; Literature Search - S.I., I.B., M.K.; Writing - S.I., I.B., M.K.; Critical Review - M.K.

Conflict of Interest: The authors have no conflicts of interest to declare.

Financial Disclosure: The authors declared that this study has received no financial support.

\section{REFERENCES}

I. Connor SE, Leung R, Natas S. Imaging of the petrous apex: a pictorial review. Br J Radiol 2008; 81: 427-35. [CrossRef]

2. Razek AA, Huang BY. Lesions of the petrous apex: classification and findings at CT and MR imaging. Radiographics 2012; 32: 15I-73. [CrossRef]

3. Radhakrishnan R, Son HJ, Koch BL. Petrous apex lesions in the pediatric population. Pediatr Radiol 2014; 44: 325-39. [CrossRef]

4. Moore KR, Harnsberger HR, Shelton C, Davidson HC. 'Leave me alone' lesions of the petrous apex. AJNR Am J Neuroradiol 1998; 19: 733-8.

5. Chapman PR, Shah R, Curé JK, Bag AK. Petrous apex lesions: pictorial review. AJR Am J Roentgenol 20ll; 196(Suppl 3): WS26-37. [CrossRef]

6. Moore KR, Fischbein NJ, Harnsberger HR, Shelton C, Glastonbury CM, White DK, et al. Petrous apex cepholoceles. AJNR Am J Neuroradiol 200l; 22: |867-7|.

7. Muckle RP, De la Cruz A, Lo WM. Petrous apex lesions. Am J Otol 1998; 19: 219-25.

8. Pisaneschi MJ, Langer B. Congenital cholesteatoma and cholesterol granuloma of the temporal bone: role of magnetic resonance imaging. Top Magn Reson Imaging 2000; II: 87-97. [CrossRef]

9. Larson TL, Wong ML. Primary mucocele of the petrous apex: MR appearance. AJNR Am J Neuroradiol 1992; 13: 203-4.

10. Ilıca AT, Hıdır Y, Bulakbașı N, Satar B, Güvenç I, Arslan HH, et al. HASTE diffusion-weighted MRI for the reliable detection of cholesteatoma. Diagn Interv Radiol 2012; 18: 153-8. 